\title{
HIV AND RISK BEHAVIOUR AMONG MEN WHO HAVE SEX WITH men in DenMark - the 2006 Sex Life Survey
}

\author{
S A Cowan (sco@ssi.dk) 1 , J Haff ${ }^{2}$ \\ 1. Statens Serum Institut, Copenhagen, Denmark \\ 2. STOP AIDS, Copenhagen, Denmark
}

\begin{abstract}
Behavioural surveys among men who have sex with men (MSM) are important for HIV surveillance. The Danish 2006 Sex Life Survey was carried out as a self administered questionnaire, which was distributed at gay venues and bars and posted on the internet. The questionnaire was completed by $3,141 \mathrm{MSM}$. We describe the methods, the respondent group and the results of the 2006 Sex Life Survey, and discuss it implications. The main finding of this survey is that $33 \%$ of the respondents have practised unsafe sex, defined as unprotected anal intercourse with one or more partners of different or unknown HIV status. In the three previous Sex Life Surveys of 2000, 2001 and 2002, this figure was between $26 \%$ and $28 \%$.
\end{abstract}

\section{Introduction}

Following a period of decreasing incidence of newly diagnosed cases of human immunodeficiency virus (HIV) infection from the early 1990s to 2000, the rate of newly detected HIV infections began to rise again in Denmark, as it did in many EU countries [1,2]. The main mode of HIV transmission in Denmark is unprotected sex between men who have sex with men (MSM). An important approach to understand the dynamics of this rising trend and contribute to evidence-based HIV prevention, is to conduct second generation surveillance - that is surveillance which combines monitoring of new HIV cases and indicators of sexual behaviour among persons in the groups at highest risk for infection [3] .

Since 2000, four Sex Life Surveys monitoring sexual behaviour and responses to HIV issues among MSM in Denmark have been carried out in cooperation between STOP AIDS - Gay Men's HIV Organization and Statens Serum Institut, with financial support from the National Board of Health. All four surveys were quantitative analyses with data collection on sexual behaviour and self-reported HIV prevalence among MSM in Denmark [4]. This paper describes the results of the most recent, fourth survey performed in 2006.

\section{Methods}

The 2006 survey was carried out between mid-August and midOctober 2006 by handing out questionnaires during the annual Copenhagen Gay Pride event and placing questionnaires in gay bars, clubs and other venues in Copenhagen and in the second largest city in Denmark, Aarhus. Questionnaires were also distributed as inserts in magazines both gay and HIV-related journals. In addition, the questionnaire was posted on several sites on the internet, both gay and HIV-related websites. This sampling method was the same as in the other Sex Life Surveys.
The questionnaire was constructed so that it would be possible to compare the results with those from earlier Danish surveys and with the outcomes of other European surveys among MSM (e.g. Gay Men's Sex Survey by Sigma Research in the United Kingdom and Barometre Gay by INVS in France). Most questions and the recall period of 12 months were identical in all four Sex Life Surveys. The questionnaire was limited to 28 questions in order to be contained within a single paper sheet.

The questions were arranged in four categories: a) demographic data/background data (age, education, residence, homo/bisexual behaviour and HIV status); b) sexual behaviour (frequency of sex, number of partners, unprotected anal sex, etc.); c) knowledge about and attitudes towards HIV and sex-related matters; d) response to various safe sex campaigns.

The internet version of the questionnaire contained exactly the same questions as the paper version, but had a number of additional pop-up double-check questions in case of answers that were inconsistent (e.g. the date of the last positive test being earlier than the year of HIV detection). Both versions were tested in a pilot study of 30 MSM contacted in gay bars in Copenhagen.

Data analysis was performed using Stata version 8. Chi-square test was used for bivariate comparisons, and multivariate logistic regression was applied to assess odds ratios (OR) and significance of independent variables for main sexual behaviour outcomes. A non-parametric test (Kruskal-Wallis) was used to compare number of partners in different groups.

When analysing the data the following definitions were used:

- Unprotected anal sex = penetrative anal sex without a condom, no distinction between insertive and receptive anal sex.

- Unsafe sex = unprotected anal sex when serostatus of the respondent is unknown, or with a partner with unknown HIV serostatus, or with a partner whose HIV serostatus is different from the perceived or known serostatus of the respondent.

In the data analyses different denominators are used, i.e. not the total number of respondents but the number of respondents who provided particular information.

Results

Demographic and background data

A total of 3,141 responses from survey participants were analysed. Of these, 2,026 (64\%) responses were obtained from 
questionnaires posted on the internet, 468 (15\%) from those handed out at the gay pride, $411(13 \%)$ from those disseminated in gay bars or saunas, and $236(8 \%)$ from those distributed via magazines.

The mean age of the respondents was 33 years (range: 15-85 years). Fifty-six percent lived in the Copenhagen Area, $27 \%$ in Aarhus, Odense or Aalborg, and 27\% outside the large cities.

Fifty-five percent had either completed, or were attending postsecondary vocational or post-secondary academic education. This level of education was high compared to the general population, but it did not differ from other surveys among MSM.

During the 12-month study period, 2,755 (88\%) of the respondents had sex with men exclusively, whereas $386(12 \%)$ had sex with both men and women (hereafter referred to as homo or bisexual behaviour, respectively). Bisexual behaviour was stated by $343(17 \%)$ of the 2,026 internet respondents, but only by $43(4 \%)$ of those 1,115 who responded to paper questionnaires. This difference can be associated with a geographical pattern, as a higher proportion of paper respondents were Copenhagen residents, and a higher proportion of internet respondents were non-Copenhagen residents. Accordingly, 88 (6\%) of respondents in Copenhagen reported bisexual behaviour, compared to 197 (18\%) of respondents outside Copenhagen $(p<0.001)$.

Two hundred thirty-one ( $8 \%$ ) of the 2,918 respondents who answered this question reported to be HIV-positive, 2,188 (75\%) to be HIV-negative, and 499 (17\%) stated that they did not know their HIV status. There was a significant difference in geographic distribution, as $10 \%$ of the respondents living in the Copenhagen area were HIV-positive whereas among those living in the rest of the country this proportion was only $4 \%(p<0.001)$. Among Copenhageners, $17 \%$ did not know their HIV-status, and among non-Copenhageners this figure was $19 \%$ (non significant).

\section{F I G U R E}

Type of anal sex partners reported in 2006 Sex Life Survey, Denmark $(n=2,932)$

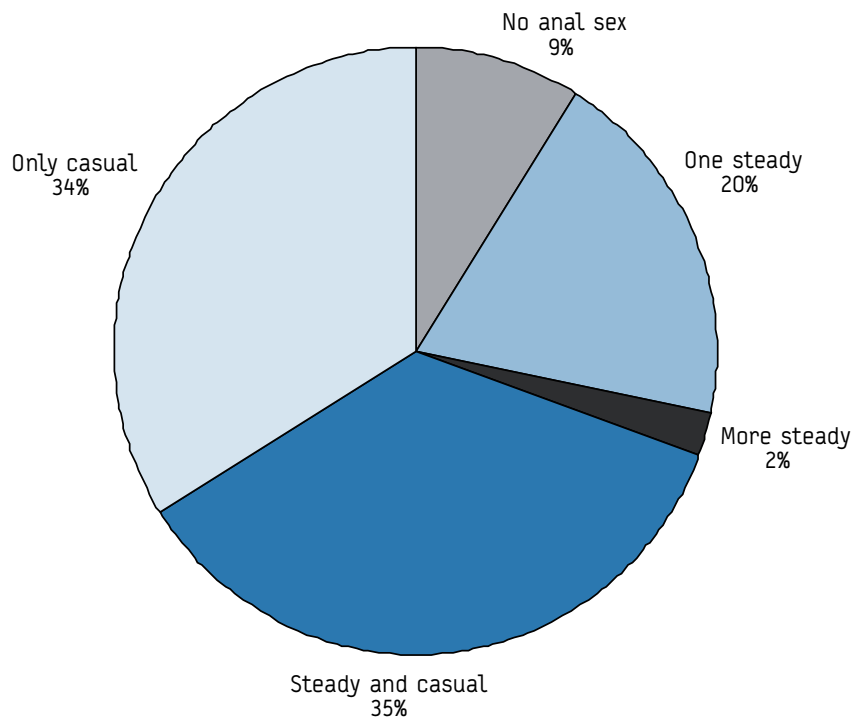

\section{Sexual behaviour}

A total of 2,836 (92\%) of the 3,095 respondents who answered this question had practised anal sex within the past 12 months. In comparison, $86 \%$ of the respondents in the Sex Life Surveys of 2000 and 2002 had practised anal sex, and the percentage was $84 \%$ in 2001 . A logistic regression analysis controlling for age, residence, HIV status and homo or bisexual behaviour showed the 2006 increase to be significant (OR=1.9, $95 \% \mathrm{Cl}=1.6-2.3, \mathrm{p}<0.001$ ), when comparing participants of the present survey to participants of any of the three former surveys.

The average number of anal sex partners reported by respondents in the 2006 survey was 9.4. In the previous three Sex Life Surveys this number was 8 . Likewise, the median has increased from two in previous years to three in $2006(p<0.001)$.

The proportion of men who had practised anal sex was the same for HIV-positive and HIV-negative men, but HIV-positive respondents had more anal sex partners (mean 17, median 6) than HIV-negative respondents (mean 8 , median 3$)(p<0.001)$. Among HIV-negatives, $180(10 \%)$ of the 1,867 respondents who stated the number of anal sex partners had more than 20 anal sex partners, while this was the case for 47 (25\%) of 186 HIV-positive respondents who stated number of anal sex partners $(p<0.001)$.

The respondents were asked to state the number of both steady and casual partners during the last 12 months. The terms "steady" and "casual" were not defined in the questionnaire, and it is unknown to what extent partners may have overlapped or succeeded each other.

Thirty-four percent had only casual partners - one or more during the last 12 months. A similar proportion, 35\%, had both steady and casual partners, whereas only $20 \%$ had one steady partner. A small group (2\%) reported more than one steady partner but no casual partners (Figure). In comparison, in the 2000 Sex Life Survey (the only one of the former surveys that distinguished between steady and casual partners), $26 \%$ of the respondents had only one steady partner, $28 \%$ had only casual partners and $29 \%$ had both steady and casual partners. A similarly small proportion (2\%) had more than one steady partner but no casual ones $(p<0.001)$.

The survey did not distinguish between insertive and receptive anal sex. When asked about unprotected anal sex, that is anal sex without condom, it was declared by $58 \%$ of the respondents.

Among HIV-positive respondents, $66 \%$ had practised unprotected anal sex, while this was the case for $55 \%$ of the HIV-negatives and for $60 \%$ of respondents who did not know their HIV status $(p=0.001)$.

Among respondents with one steady partner, $73 \%$ had practised unprotected anal sex. This proportion was $71 \%$ among respondents with both steady and casual partners, 50\% among respondents with only casual partners, and $51 \%$ among respondents with more than one steady, but no casual partners $(p<0.001)$. Compared to the 2000 Sex Life Survey, the respondents who had only casual partners had a significantly higher proportion of unprotected anal sex in $2006(p<0.001)$. For the respondents who had both steady and casual partners, the increase in the proportion of those who had unprotected anal sex was only marginally significant $(p=0.06)$. Among respondents who had only one steady partner, there was no 
difference in the proportion of those who had unprotected anal sex between the two Sex Life Surveys.

Among homosexual MSM, 59\% had practised unprotected anal sex, compared with $48 \%$ of bisexual men $(p<0.001)$.

Among respondents under 30 years of age, $62 \%$ had practised unprotected anal sex, compared with $55 \%$ of respondents aged 30 years or older $(p<0.001)$. When stratified by number and type of anal sex partners, there was no difference between the age groups, if respondents had only steady partners. Among respondents who had both steady and casual partners, 301 of 404 (75\%) respondents less than 30 years old had unprotected anal sex, compared to 341 out of $499(68 \%)$ respondents aged 30 years or more $(p=0.04)$. Among respondents who had only casual partners, 195 of 360 (54\%) respondents less than 30 years old had unprotected anal sex compared to 242 of 556 (46\%) respondents aged 30 years or more $(p=0.02)$.

Education level or place of residence was not of significant importance with regard to having practiced unprotected anal sex.

Among men who had only had one anal sex partner during the last 12 months (casual or steady), $66 \%$ had unprotected anal sex. Among men who had two or more partners, the fraction of those who had unprotected anal sex ranged from $55 \%$ (2-5 partners) to $71 \%$ (>20 partners).

\section{Unsafe sex}

In this survey, unsafe sex is defined as unprotected anal sex when serostatus of the respondent is unknown, or with a partner with unknown HIV serostatus, or with a partner whose HIV serostatus is different from the perceived or known serostatus of the respondent. Of the respondents, 33\% stated that they had practised unsafe sex at least once during the last 12 months.

To assess possible predictors of unsafe sex, a multivariate logistic regression analysis was carried out. Six factors turned out to be independently associated with unsafe sex: Number of anal

\section{T A B L E 1}

Frequency and odds ratios (OR) for independent variables which were significant predictors of unsafe sex in a logistic regression analysis; 2006 Sex Life Survey, Denmark

\begin{tabular}{|c|c|c|}
\hline & $\mathrm{N}(\%)^{*}$ & $\begin{array}{l}\text { Multivariate } \\
\text { OR }\end{array}$ \\
\hline $\begin{array}{l}\text { Anal sex partners }<3,>0 \\
\text { Anal sex partners } \geq 3\end{array}$ & $\begin{array}{l}1,046(36 \%) \\
1,627(55 \%)\end{array}$ & $\begin{array}{l}1 \\
4.4(3.50-5.62)\end{array}$ \\
\hline $\begin{array}{l}\text { HIV-negative }{ }^{\star \star} \\
\text { HIV-positive }\end{array}$ & $\begin{array}{l}2,188(75 \%) \\
231(8 \%)\end{array}$ & $\begin{array}{l}1 \\
3.1(2.20-4.37)\end{array}$ \\
\hline $\begin{array}{l}\text { High risk perception }{ }^{\star \star \star} \\
\text { Low risk perception }\end{array}$ & $\begin{array}{l}2,569(87 \%) \\
377(13 \%)\end{array}$ & $\begin{array}{l}1 \\
2.67(1.98-3.61)\end{array}$ \\
\hline $\begin{array}{l}\text { Age } \geq 30 \text { years } \\
\text { Age }<30 \text { years }\end{array}$ & $\begin{array}{l}1,673(60 \%) \\
1,124(40 \%)\end{array}$ & $\begin{array}{l}1 \\
1.7(1.32-2.06)\end{array}$ \\
\hline $\begin{array}{l}\text { Post-secondary vocational or academic } \\
\text { education } \\
\text { Primary and secondary education }\end{array}$ & $\begin{array}{l}1,526(55 \%) \\
1,266(45 \%)\end{array}$ & $\begin{array}{l}1 \\
1.3(1.04-1.61)\end{array}$ \\
\hline $\begin{array}{l}\text { Frequency of sex: once a month or less } \\
\text { often } \\
\text { Frequency of sex: several times a month } \\
\text { or more often }\end{array}$ & $\begin{array}{l}1,041(33 \%) \\
2,100(67 \%)\end{array}$ & $\begin{array}{l}1 \\
1.3(1.01-1.61)\end{array}$ \\
\hline
\end{tabular}

OR: odds ratio

* Number and proportion (\%) of respondents who answered the question concerned

${ }_{\star *}$ Respondents with unknown HIV status were excluded from the analysis *** High risk perception: attributing great or very great risk of unprotected anal sex; low risk perception: attributing low or no risk of unprotected anal sex sex partners, HIV status, risk perception, age, education level and frequency of having sex (Table 1). There was no association between unsafe sex and residence, homo- or bisexual behaviour, or whether the questionnaire was submitted online or on paper, neither in bivariate nor multivariate analyses. Having had a new anal sex partner within the last 12 months was a significant predictor for unsafe sex in the bivariate analyses, but not in the multivariate analysis.

The number of partners was the strongest predictor of unsafe sex; the probability of having had unsafe sex ranged from $17 \%$ in men with one partner to $58 \%$ in men with more than 20 partners $(p<0.001)$.

HIV status was also a strong predictor. In a bivariate analysis, $49 \%$ of HIV-positive men had practised unsafe sex compared to $25 \%$ of HIV-negative men $(p<0.001$ ). Men who did not know their HIV status were the group among whom unsafe sex was practised by the biggest proportion (60\%). This is due to the fact that all unprotected anal sex in this group was considered unsafe sex.

In the three earlier Sex Life Surveys, the proportions of respondents reporting unsafe sex ranged from $26 \%$ to $28 \%$. As the populations in the four surveys differed in demographic composition, it is not possible to make a direct comparison. However, a multiple regression analysis shows that the proportion of respondents who practised unsafe sex had increased by $20-30 \%$ since the 2000 , 2001 and 2002 surveys, when controlling for age, HIV status, education, number of partners, and frequency of sex. Unsafe sex was further stratified by discordant/unknown status. Table 2 presents the different strata of safe/unsafe sexual behaviour.

\section{Risk perception and assessment of the risk of HIV transmission}

Unprotected anal sex is known to be the most risky sexual practice for HIV transmission. Respondents were asked to state their perception of the risk of HIV transmission when practising anal sex with and without condom use, and with and without ejaculation, respectively. The majority $(88 \%)$ stated that the risk of HIV transmission during anal sex without a condom and with ejaculation inside the partner was "risky" or "very risky". As noted above, perceiving the risk as "low" or "not risky" was a predictor of having practised unsafe sex. Whether the respondents perceived the practices to be risky or not, we examined the individual answers according to the level of risk assigned to the different anal sex practices (with and without a condom and with and without ejaculation inside the partner). The way the respondents

\section{T A B L E 2}

Overview of respondents' sexual behaviour within the last 12 months; 2006 Sex Life Survey, Denmark

\begin{tabular}{|l|c|}
\hline Behaviour & $\begin{array}{c}\text { Number of } \\
\text { respondents (\%) }\end{array}$ \\
\hline No anal sex & $231(8 \%)$ \\
\hline Only protected anal sex & $974(35 \%)$ \\
\hline Unprotected anal sex with concordant partners & $663(24 \%)$ \\
\hline $\begin{array}{l}\text { Unprotected anal sex without knowing own and/or } \\
\text { partners' HIV status }\end{array}$ & $737(28 \%)$ \\
\hline Unprotected anal sex with discordant partners & $187(5 \%)$ \\
\hline Total (who stated HIV status and sexual behaviour) & $\mathbf{2 , 7 9 2 ( 1 0 0 \% )}$ \\
\hline
\end{tabular}


ranked the risk levels was used as a marker for knowledge of HIV transmission risk, so that anal sex without a condom with ejaculation inside the partner had to be ranked as more risky than without ejaculation, which in turn had to be ranked more risky than anal sex with a condom. Ninety-seven percent ranked the levels of risk satisfactorily.

\section{HIV testing}

Seventy seven percent of the respondents had undergone HIV testing one or more times in their lifetime.

Among respondents who stated the year of the last test, $36 \%$ had been tested in 2006. The questionnaire was distributed in the period August-October, so the answers could not reflect test activity in a full year. When including respondents whose last test had taken place in 2006 or 2005 (i.e. max 22 months ago), the figure was $59 \%$. The corresponding figure was $51 \%$ in 2001 and $50 \%$ in 2002 survey (data were not available in 2000 survey) $(p<0.001)$.

There was no difference in whether an individual had been practising unsafe sex during the last 12 months or not, in relation to whether he had ever been tested. However, among men who had practised unsafe sex during the last 12 months and were not HIVpositive, $48 \%$ had been tested in 2006 or 2005 , while this was the case for only $43 \%$ of those who had not practised unsafe sex and were not HIV-positive. Two thirds of the respondents who had unsafe sex also stated how often they had it. Among those, testing frequency did not reflect risk taking; respondents who had unsafe sex once or twice during the last 12 months were more often tested recently than respondents who had unsafe sex 3-10 times, who, in turn, were tested recently more often than respondents who had unsafe sex more than 10 times during the last 12 months. This trend was, however, only marginally significant $(p=0.06)$

\section{Disclosure and condom use with a new partner}

In the course of the last 12 months, $66 \%$ of respondents had practised anal sex with a new partner with whom they had not previously had anal sex. Of these, $22 \%$ did not use a condom during the most recent occasion they had anal sex with a new partner, i.e. they had practiced unprotected anal sex.

Overall, $31 \%$ of those who had anal sex with a partner with whom they had not previously had anal sex informed their partner of their HIV status (disclosure) prior to having sex (only $1 \%$ disclosed it after sex). The same number of men were informed about their partner's status (received disclosure). There was an almost total overlap in these two groups, indicating that people either practised mutual disclosure or that neither of them disclosed.

\section{T A B L E 3}

Disclosure of HIV status and condom use; 2006 Sex Life Survey, Denmark

\begin{tabular}{|l|c|c|}
\hline Disclosure/condom & $\begin{array}{c}\text { Number of } \\
\text { respondents }\end{array}$ & $\begin{array}{c}\text { Proportion of } \\
\text { total (\%) }\end{array}$ \\
\hline No disclosure, no condom & 196 & 11 \\
\hline Condom but no disclosure & 975 & 56 \\
\hline Disclosure but no condom & 171 & 10 \\
\hline Both disclosure and condom & 394 & 23 \\
\hline $\begin{array}{l}\text { Total (who provided this information in } \\
\text { the questionnaire) }\end{array}$ & 1,736 & 100 \\
\hline
\end{tabular}

Forty-nine percent of the respondents who had not been using a condom last time they practised anal sex with a new partner disclosed their HIV status, compared with $30 \%$ of those who did use a condom. As shown in Table 3, 11\% did not use condoms and did not disclose their HIV status the last time they practiced sex with a new partner, matching the study definition of unsafe sex.

Nearly half (48\%) of the respondents had met their new partner on the internet. This figure was higher among internet respondents (57\%) and lower (33\%) among those who submitted paper questionnaires $(p<0.001)$. The internet, bars/discotheques and saunas/sex clubs constituted a total of $79 \%$ of the answers to the question on where the respondents had met their latest new partners, regardless of the questionnaire source.

\section{Gay magazines, venues and websites}

Sixty percent read gay magazines, $82 \%$ used websites for homosexuals and $74 \%$ frequented gay venues. Fifty nine percent of the respondents used both gay venues and websites, $15 \%$ used venues exclusively, $12 \%$ only websites, and $14 \%$ used none of these.

\section{Discussion}

This survey included 3,141 MSM representing $6.4 \%$ of the estimated 50,000 MSM in Denmark who in turn constitute $2.5 \%$ of the adult male population (aged $15-80$ years) [5].

It is not possible to calculate a response rate, nor can it be known if the MSM who were not reached with the questionnaire or who chose not to answer, differ from the respondents in demographical or behavioural parameters. Even though the large number of internet respondents facilitated the inclusion of MSM outside the big cities, it is quite possible that MSM who answered the questionnaire represent a more outgoing and sexually active fragment of the Danish MSM population.

In this survey, only $20 \%$ of the respondents appeared to be practising a monogamous sex life with one steady partner, whereas the majority had both steady and casual partners or only casual partners. The extensive change of partners facilitates the spread of sexually transmitted infections, including HIV infection.

The main finding of this survey is that $33 \%$ of the respondents have practised unsafe sex, defined as unprotected anal intercourse with one or more partners of different or unknown HIV status. In the three previous Sex Life Surveys of 2000, 2001 and 2002, this figure was between $26 \%$ and $28 \%$, indicating an increase of $20-30 \%$, when controlled for population differences. There is no perfect way of dealing with differences when trying to compare different convenience samples, but controlling for factors that were shown to influence the risk of unsafe sex in bivariate analyses of both the present and the former Sex Life Surveys goes some way to overcome this issue. Furthermore, the same logistic regression analysis showed no difference in unsafe sex between the years 2000, 2001 and 2002, when controlling for the same factors. The fact that more respondents were recruited via the internet did not have an independent impact, when different rates of unsafe sex were analysed.

Several studies in other European countries [6-8] have reported increased frequency of unsafe sex among MSM in the early 2000s. However, during the recent years, unsafe sex levels seem to have 
stabilised among MSM in some countries [9]. The increase in proportion of MSM practising unsafe sex between the 2002 and the 2006 Sex Life Surveys could have taken place at any time during this four-year period, and only repeated surveys will show if the trend in Denmark is still increasing.

The fact that MSM who are aware of their positive HIV status reported the highest levels of unsafe sex is problematic, but it mirrors recent findings elsewhere $[6,10,11]$.

Among HIV-positive respondents, $66 \%$ had unprotected anal sex, but the proportion of those HIV-positive respondents who had unprotected anal sex with partners they did not know to be seroconcordant (of same HIV status) was $49 \%$, suggesting that some amount of serosorting (the practice of having unprotected anal intercourse with a partner believed to be of the same HIV status $[12,13])$ among HIV-positive MSM takes place. The difference among HIV-negative respondents who have unprotected anal sex (55\%) and HIV-negative respondents who have unsafe sex $(25 \%)$ is even bigger. Whether this is due to HIV-negative MSM practicing active serosorting, or it is merely due to the more easy access that HIV-negative MSM have to seroconcordant partners, is not known. Serosorting among men who perceive themselves to be HIV-negative is only of value if both partners have had no risk of becoming infected since last negative test, and several studies have demonstrated that relying on negative serosorting with casual partners often leads to HIV transmission $[10,14,15]$.

An even stronger predictor of unsafe sex than HIV positivity was the number of anal sex partners. This issue is recurrent in all the previous

Sex Life Surveys as well as in surveys in other countries [16]. In this context it is noteworthy that the average number of anal sex partners has increased since the 2002 Sex Life Survey.

The present survey does not offer an explanation as to why the numbers of partners, unprotected anal intercourse and unsafe sex are increasing. Among the reasons suggested by researchers in the field are treatment optimism, "safe sex fatigue", and the absence of the deterring effect of friends and lovers who are ill [17]. Especially the younger generations have begun their sex life in this day and age when HIV is no longer considered a threat of early death. This may partly explain why in our study younger MSM had unsafe sex more often than the older MSM.

It may be that the findings reflect a general tendency towards a more liberal and uninhibited sex life following a couple of decades of caution. Men's sex life is influenced by other factors than those that have to do with risk and HIV. An additional reason for increase of unprotected anal sex could be a switch from risk avoidance towards risk management strategies, e.g. serosorting.

Although $33 \%$ of people practising unsafe sex is a high percentage, there are still many MSM who exclusively had safe sex. The respondents were not asked about the number of partners with whom they had practised safe sex, or how many times.

The survey included assessment of the risk of HIV transmission in the case of unprotected anal intercourse with ejaculation in the partner. However, the participants were not asked to assert whether they practiced insertive or receptive anal sex or both, so some respondents could have interpreted the question in light of their own practices, and not, as intended, as the general possibility of transmitting HIV by ejaculating into the partner, i.e. transmission from the insertive partner to the receptive one.

Men who had a low estimation of the risk were more likely to have practised unsafe sex than men who estimated that the risk was high. However, on the basis of this survey results, it cannot be determined whether individuals choose to practise unsafe sex because they estimate the risk to be low, or they may be rationalising - after having practised unsafe sex - that the risk might not be that high after all.

MSM with an education level corresponding to post-secondary vocational or academic education had a lower risk of having unsafe sex than the less well educated MSM in this survey. The level of knowledge regarding safe sex practice was very high regardless of educational level, so this finding is surprising. Also, education level has not been a significant predictor in the former Sex Life Surveys.

Finally, the frequency of sex was an independent predictor of unsafe sex, but not as strong as the number of anal sex partners.

The overall HIV prevalence in the study was $8 \%$, with a higher HIV prevalence among residents of the capital (10\%) than among respondents from the rest of the country (4\%). The wide use of the internet questionnaire in the 2006 Sex Life Survey has contributed to a larger proportion of responses from internet respondents living outside of Copenhagen in the 2006 survey than in the previous three Sex Life Surveys. Consequently, the overall HIV prevalence was lower than in the past surveys when it ranged between 10 and $11 \%$. The prevalence estimate obtained by using data from the national surveillance system and the Danish HIV Cohort [18] is $5 \%$. The result of our study is in line with this, taking into account that the survey still contained a disproportionately big fraction of Copenhageners with higher HIV prevalence than in the rest of the country. However, this can not be quantified, since the population distribution of MSM in Denmark is not known. Furthermore, the very high prevalence among the 61 respondents who had received the questionnaire as an insert in a HIV related magazine contributed to increase the overall prevalence.

Practically all respondents ranked different anal sex practice risks in the right order. This indicates a very high level of awareness concerning risky sex behaviours. Also in 2000, respondents demonstrated a good knowledge of risky sex behaviour. The awareness level is thus still high, a fact that may be ascribed to earlier information campaigns.

Future prevention initiatives must not only aim at maintaining this high level but also address the fact that unsafe sex is taking place despite the widespread and thorough knowledge of risks.

More than three-fourths of the respondents had undergone HIV testing one or more times in their lifetime. In other European countries, this figure varies between $50 \%$ and $80 \%$ [19]. Half of the respondents who had practised unsafe sex (and who had not previously been tested positive) had been tested in 2006 or 2005 , implying that half of those respondents who could in principle have been infected within the last 12 months had not been tested within this period [12]. This was the case for a somewhat smaller number of respondents who - according to their questionnaire replies - had run no risk of HIV infection. From a prevention perspective, the point is not to make as many people as possible take the test, but 
to make the relevant people take the test - those who have run a risk of being infected.

Two thirds of the respondents had anal sex with a new partner during the last 12 months, confirming the impression of a high partner turnover. Eleven percent did not know the HIV status of their new partner, but they still did not use a condom at the latest intercourse with the new partner. This method of assessing unsafe sex (at the last anal intercourse) adds an additional level to the measure of unsafe sex during the last 12 months.

Nearly half of the respondents had met their new partner via internet. From a prevention perspective, it is relevant that the internet is such a popular contact place. Gay venues such as bars/discos and sauna/sex clubs were used by two thirds of the respondents, and more than half of the respondents used both gay venues and gay websites. The proportion of respondents who used only venues or websites, or neither of these were much smaller (15\%, $12 \%$ and $14 \%$, respectively. In the light of both internet and gay venues playing a considerable role in the social and sexual life of MSM, preventive efforts focused on both these information media should make it possible to reach a large number of this population.

HIV among MSM is still a serious problem in Denmark and in the rest of Europe, and will continue to be so as a considerable proportion of MSM practise unsafe sex. The present survey demonstrates a high level of knowledge in this target group. However, knowledge is not enough to ensure safe sex practices, and the frequency of unsafe sex among MSM seems to be increasing. This finding has been used in safer sex campaigns conducted by STOP AIDS - Gay Men's HIV Organization, who tailor campaigns to influence attitudes and actions and not just knowledge about HIV transmission [20]. Monitoring developments and trends in the sexual behaviour among MSM is thus important, not only on a national level, but also in a European and a global context. Hopefully, the Danish Sex Life Survey will be continued regularly in the future, and behavioural surveys among MSM on a European scale will be undertaken.

\section{References}

1. Hamers FF, Downs AM. The changing face of the HIV epidemic in western Europe: what are the implications for public health policies? Lancet. 2004;364(9428):83-94

2. Nardone A. Transmission of HIV/AIDS in Europe continuing. Euro Surveill. 2005;10(47):pii=2837. Available from: http://www.eurosurveillance.org/ ViewArticle. aspx? ArticleId $=2837$

3. Joint United Nations Programme on HIV/AIDS (UNAIDS), World Health Organization (WHO). Second generation surveillance for HIV: The next decade. Available from: http://www.who.int/hiv/pub/surveillance/en/cds_edc_2000_5. pdf

4. Haff J, Cowan S. [Sex life Survey 2006 HIV and sex among men who have sex with men]. Copenhagen: STOP AIDS and Statens Serum Institut; 2007. [in Danish]

5. Cowan SA, Smith E. [Incidence of HIV/AIDS in Denmark, 1990-2005]. Ugeskr Laeger. 2006;168(23):2247-52.

6. Bezemer D, de WF, Boerlijst MC, van SA, Hollingsworth TD, Prins M, et al. A resurgent HIV-1 epidemic among men who have sex with men in the era of potent antiretroviral therapy. AIDS. 2008;22(9):1071-7.

7. Gebhardt M. Recent trends in new diagnoses of HIV infections in Switzerland: probable increase in MSM despite an overall decrease. Euro Surveill. 2005;10(49):pii=2850. Available from: http://www.eurosurveillance.org/ ViewArticle. aspx? ArticleId $=2850$

8. Marcus U, Voss L, Kollan C, Hamouda O. HIV incidence increasing in MSM in Germany: factors influencing infection dynamics. Euro Surveill. 2006;11(9):pij=645. Available from: http://www.eurosurveillance.org/ ViewArticle.aspx?ArticleId $=645$
9. Elford J, Bolding G, Sherr L, Hart G. High-risk sexual behaviour among London gay men: no longer increasing. AIDS. 2005;19(18):2171-4.

10. Williamson LM, Dodds JP, Mercey DE, Hart GJ, Johnson AM. Sexual risk behaviour and knowledge of HIV status among community samples of gay men in the UK. AIDS. 2008;22(9):1063-70.

11. Dodds JP, Johnson AM, Parry JV, Mercey DE. A tale of three cities: persisting high HIV prevalence, risk behaviour and undiagnosed infection in community samples of men who have sex with men. Sex Transm Infect. 2007;83(5):392-6.

12. Mackellar DA, Valleroy LA, Behel S, Secura GM, Bingham T, Celentano DD, et al. Unintentional HIV exposures from young men who have sex with men who disclose being HIV-negative. AIDS. 2006;20(12):1637-44.

13. Pinkerton SD, Galletly CL. Reducing HIV transmission risk by increasing serostatus disclosure: a mathematical modeling analysis. AIDS Behav. 2007;11(5):698-705.

14. Williamson LM, Hart GJ. HIV prevalence and undiagnosed infection among a community sample of gay men in Scotland. J Acquir Immune Defic Syndr. 2007;45(2):224-30.

15. Eaton LA, Kalichman SC, Cain DN, Cherry C, Stearns HL, Amaral CM, et al. Serosorting sexual partners and risk for HIV among men who have sex with men. Am J Prev Med. 2007;33(6):479-85.

16. Brewer DD, Golden MR, Handsfield HH. Unsafe sexual behavior and correlates of risk in a probability sample of men who have sex with men in the era of highly active antiretroviral therapy. Sex Transm Dis. 2006;33(4):250-5.

17. Elford J. Changing patterns of sexual behaviour in the era of highly active antiretroviral therapy. Curr Opin Infect Dis. 2006;19(1):26-32.

18. Lohse N, Hansen AB, Jensen-Fangel S, Kronborg G, Kvinesdal B, Pedersen C, et al. Demographics of HIV-1 infection in Denmark: results from the Danish HIV Cohort Study. Scand J Infect Dis. 2005;37(5):338-43.

19. Mikolajczak J, Hospers HJ, Kok G. Reasons for not taking an HIV-test among untested men who have sex with men: an Internet study. AIDS. Behav 2006;10(4):431-5.

20. STOP AIDS - the gay men's HIV organisation. [Campaign information]. Available from: http://www.stopaids.dk/nyheder/kampagner.html [in Danish]

This article was published on 27 November 2008.

Citation style for this article: Cowan SA, Haff J. HIV and risk behaviour among men who have sex with men in Denmark - the 2006 Sex Life Survey. Euro Surveill. 2008:13(48):pii=19050. Available online: http://www.eurosurveillance.org/ViewArticle. aspx?ArticleId $=19050$ 